DNA methylation has been demonstrated in $T$ and $B$ cells from patients with SLE. The aim of the study was to analyse several methylation-sensitive genes in B cells from SLE patients.

Method Samples were B cell-enriched from the blood of seven patients with SLE and six healthy controls (HCs). Five cellular genes and five endogenous retroviruses expressed in lymphocytes were sought. Promoter methylation status was evaluated using MS-PCR and bisulfite sequencing. Expression was evaluated by real-time PCR. B cells were stimulated with anti-IgM antibodies or incubated with PD98059, a DNMTs inhibitor.

Results Promoter methylation status revealed three groups: a demethylated group (CD5, CD70, Pax5, Syk), a methylated group (HERV-E 4.1, HERV-E 7p22, HERV-E 17q21) and a hypomethylated group at the basal level and methylated after IgM stimulation in HCs but not in SLE B cells (HERVCD5, HRES-1). The authors provide further evidence that production of interleukin 6 abrogates the ability of SLE B cells to methylate promoters from group 3 upon stimulation. This effect is reversed with a blocking antibody. Stimulation increased group 3 expression in SLE B cells. Utilisation of the DNA methylation inhibitor PD98059 in HC B cells is associated with group 2 and 3 overexpression.

Conclusion This study shows engagement of the BCR silence hypomethylated endogenous retrovirus and that this mechanism is impaired in SLE B cells. This derepression could in turn promote the expression of endogenous retrovirus and then promote B cell autoreactivity.

\title{
A84 ENDOGENOUS RETROVIRUS CONTROL IS IMPAIRED IN B CELLS FROM PATIENTS WITH SYSTEMIC LUPUS ERYTHEMATOSUS
}

Y Renaudineau, C Le Dantec, J-0 Pers, P Youinou EA 2216 "Immunologie et Pathologie " and IFR 148 ScInBioS, Université de Brest and Université Européenne de Bretagne, Brest, France

10.1136/ard.2010.129619r

Purpose Systemic lupus erythematosus (SLE) is a heterogenous autoimmune disease of unknown origin. A defect in 